\title{
Imaging Biologically Induced Mineralization in Fully Hydrated Flow Systems
}

\author{
Logan Schultz,, Betsey Pitts,, ${ }^{1}$ Andrew C. Mitchell,, ${ }^{12}$ Alfred B. Cunningham, ${ }^{1}$ \\ and Robin Gerlach ${ }^{1}$ * \\ ${ }^{1}$ Center for Biofilm Engineering, P.O. Box 173980, Montana State University, Bozeman, MT 59717 \\ ${ }^{2}$ Institute of Geography and Earth Sciences, Aberystwyth University, SY23 3DB, UK \\ * robin_g@biofilm.montana.edu
}

\section{Introduction}

A number of proposed technologies involve the controlled implementation of biologically induced carbonate mineral precipitation in the geologic subsurface. Examples include the enhancement of soil stability [1], immobilization of groundwater contaminants such as strontium and uranium [2], and the enhancement of oil recovery and geologic carbon sequestration via controlled permeability reduction [3]. The most significant challenge in these technologies remains to identify and better understand an industrially, environmentally, and economically viable carbonate precipitation route.

One of the most promising routes is ureolytic biomineralization, because of the ample availability of urea and the controllable reaction rate. In this process, ureolytic bacteria hydrolyze urea, leading to an increase in $\mathrm{pH}$. In the presence of calcium, this process favors the formation of solid calcium carbonate, as illustrated in the following equations:

$$
\begin{gathered}
\mathrm{CO}\left(\mathrm{NH}_{2}\right)_{2}+\mathrm{H}_{2} \mathrm{O} \rightarrow \mathrm{NH}_{2} \mathrm{COOH}+\mathrm{NH}_{3} \\
\rightarrow 2 \mathrm{NH}_{3}+\mathrm{CO}_{2} \quad \text { (Urea hydrolysis) } \\
2 \mathrm{NH}_{3}+2 \mathrm{H}_{2} \mathrm{O} \leftrightarrow 2 \mathrm{NH}_{4}^{+}+2 \mathrm{OH}^{-} \quad \text { (pH increase) } \\
\mathrm{CO}_{2}+2 \mathrm{OH}^{-} \leftrightarrow \mathrm{CO}_{3}^{2-}+\mathrm{H}_{2} \mathrm{O} \\
(\text { Carbonate ion formation) } \\
\mathrm{CO}_{3}^{2-}+\mathrm{Ca}^{2+} \leftrightarrow \mathrm{CaCO}_{3} \text { (solid) } \\
(\text { Precipitation is favored at high } \mathrm{pH})
\end{gathered}
$$

This process relies on molecular-level chemical and biological processes that must be better understood for large-scale implementation.

Researchers at the Center for Biofilm Engineering at Montana State University (USA) and Aberystwyth University (UK) have conducted several biomineralization experiments in simulated porous media reactors. Microscopy has proven to be one of the most useful analytical tools in these studies, providing the ability to non-invasively visualize, differentiate, and quantify the various components, including the cells, cell matrix, and mineral precipitates. Because of the possibility of real-time observation and the lack of dehydration artifacts, microscopy has been tremendously useful for elucidating the temporal and spatial relationships of these components.

\section{Methods and Materials}

The soil bacterium Sporosarcina pasteurii was used to induce ureolysis. For each study, the flow reactor was inoculated with a culture of $S$. pasteurii, and the cells were allowed to attach for 1.5 hours before flow was started. The flow medium, consisting of nutrient broth, urea, and dissolved calcium, was $\mathrm{pH}$-adjusted to 6.0 to avoid immediate mineral precipitation.

A Nikon SMZ 1500 stereo microscope was used to provide a view of the biomineralized reactor and to quantify the volume fraction of precipitates within the pore networks. A Leica TCS-SP2 AOBS confocal laser scanning microscope (CLSM) was used to evaluate the micro-scale interaction between the crystalline surfaces and the biofilm. Flow medium was collected at the outlet of the reactor and analyzed for $\mathrm{Ca}, \mathrm{NH}_{4}{ }^{+}$, and $\mathrm{pH}$. This article discusses some techniques and examples of imaging biomineralization in flow reactors with these tools.

\section{Results}

Stereo Microscopy. To characterize the spatial and temporal development of precipitates in a network of pores, the stereo microscope was placed over the reactor system (Figure 1).

Throughout the 15-hour experiment, images of one region near the inlet were taken at regular time intervals, allowing for a time-lapse video of the mineral growth to be constructed. This video provided insight into nucleation and precipitation kinetics. At the end of the experiment, when the reactor had been terminally plugged, images were taken along the length of the reactor. These images, displayed in Figure 2 below, highlight a gradient in mineral size from inlet to outlet.

From images, such as those in Figure 2, quantitative data about the process can be obtained. Using ImageJ software [4], the precipitates in each field of view were counted and measured. Subsequent X-ray diffraction analysis of the precipitates showed that calcite was the predominant mineral. Assuming the precipitates to be the density of calcite $\left(2.71 \mathrm{~g} / \mathrm{cm}^{3}\right)$ and spherical in shape, the total amount of calcium immobilized within the reactor was estimated to be $49.1 \mathrm{mg}$. Knowing the chemical composition of the inlet and outlet, in addition to the fluid flow rate, the total amount of calcium immobilized was estimated to be $82 \mathrm{mg}$ based on an effluent mass balance. Because the image-analysis method did not account for the smallest precipitates (that is, less than approximately 0.02 $\mathrm{mm}$ diameter) or the entirety of the densely precipitated inlet region (see Figure 2a), this slightly low estimate makes sense. Our analysis suggests, though, that quantitative image-analysis approaches can be effective for reactors in which image clarity is not affected by large numbers of precipitates.

A solubility constant of each measured crystal was estimated for the given molar surface area, and the crystal solubility was calculated for each region of the reactor, based on relationships outlined by Stumm et al. [5] and Mitchell and Ferris [2]. The results shown in Figure 3 suggest that the crystal 

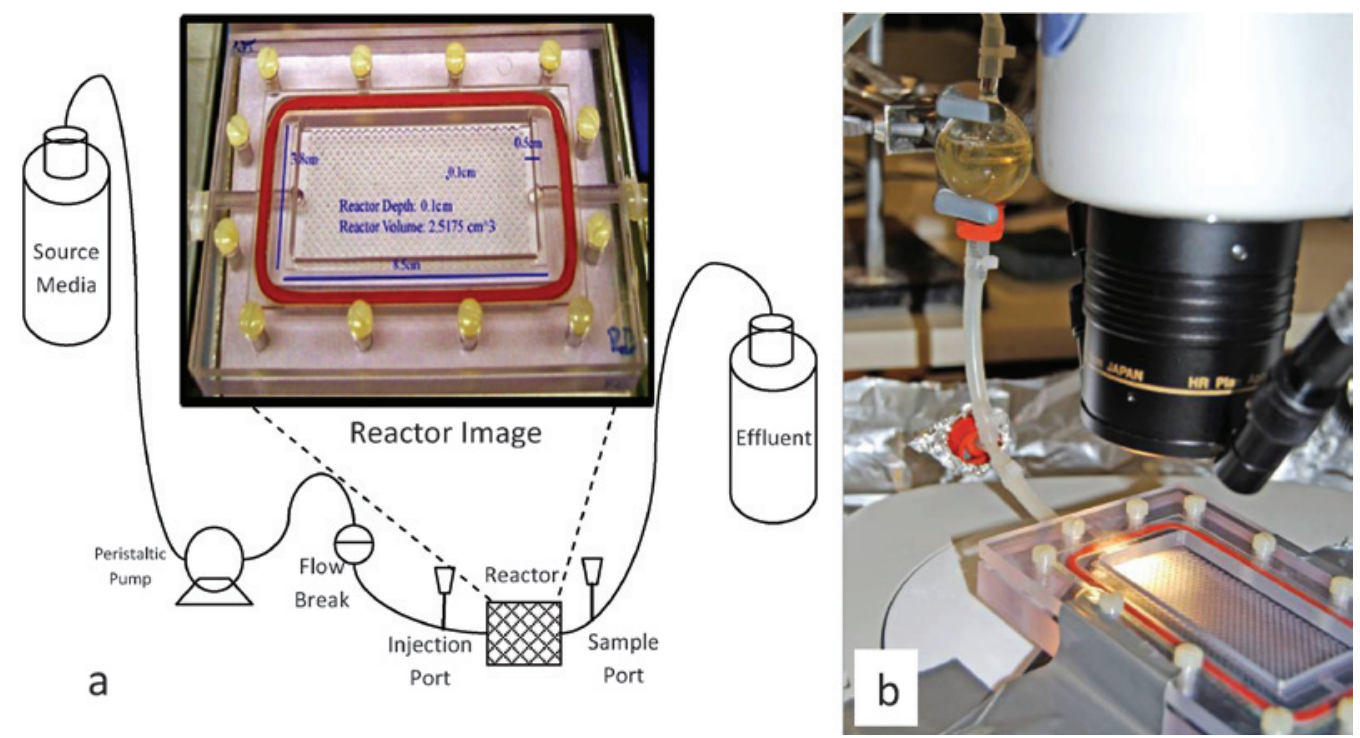

induced mineralization processes and aided our ability to understand and ultimately control the overall process. Glass capillary flow systems, previously used in our laboratory, were used for this purpose (Figure 4) [6]. The 0.17 $\mathrm{mm}$ thick glass walls allow for non-invasive CLSM. The Leica TCS-SP2 AOBS confocal microscope employing a Leica $63 \times$ water immersion lens (2 $\mathrm{mm}$ working distance) was used. Imaris image-analysis software (Bitplane Scientific Software, St. Paul, MN) was used to compile the $z$-stacks into 3-dimensional images.

After the system was run

Figure 1: (a) Schematic of the 2-dimensional reactor with a network of $1 \mathrm{~mm}$ pores. Flow moved from left to right across the system. The bacteria were introduced through the injection port, and the effluent samples were extracted through the sample port for chemical and biological analyses. (b) Photograph of the reactor under observation by the Nikon SMZ 1500 stereo microscope during biomineralization experiment.



Figure 2: Selected stereomicroscopy images taken from above the reactor and focused on mineral formation on the interior surface of the glass cover slip. (a) Inlet region showing the greatest degree of biomineralization, (b) $2 \mathrm{~cm}$ into the reactor, (c) $5 \mathrm{~cm}$ into the reactor, and (d) outlet region (approx. $8 \mathrm{~cm}$ into the reactor). Note the size gradient from inlet to outlet. Images such as these provided the data for size, density, and solubility calculations as a function of reactor location and retention time.

solubility increased with distance into the porous media reactor because of the larger free surface energy of the smaller crystals.

Confocal Laser Scanning Microscopy (CLSM). The large-scale processes observed by stereo microscopy and chemical analysis of the flow medium at the outlet of the reactor are ultimately dictated by processes that occur on a much smaller scale, demanding the ability to visualize microbe-mineral interactions on a smaller scale. Confocal laser scanning microscopy provided higher-resolution differentiation of the components involved in the biologically to differentiate the various components. In the first study, SYTO-11 and C2-dichlorotriazine (C2d) (Invitrogen, Carlsbad, CA) were used. C2d (red) is expected to bind to alcohols and polysaccharides and thus reveal the compounds most likely associated with the extracellular matrix; SYTO-11 (green) is a nucleic acid stain that is expected to bind to bacterial cells.

Figure 5 shows several images of cells and extracellular matrix surrounding what appear to be mineral precipitates (calcite). The cells appear to be attached preferentially to the calcite surface over the glass surface. Figure 5 a displays a stack of CLSM images at low magnification. The image spans the whole width $(1 \mathrm{~mm})$ of the capillary flow cell and shows clusters of cells and matrix surrounding dark areas (filled with calcite mineral). Figure $5 \mathrm{~b}$ shows a three-dimensional reconstruction of clusters of bacteria and extracellular polymeric substances, which, as seen in Figures $5 \mathrm{c}$ and $5 \mathrm{~d}$, surround areas without fluorescence suggesting that calcium carbonate precipitates

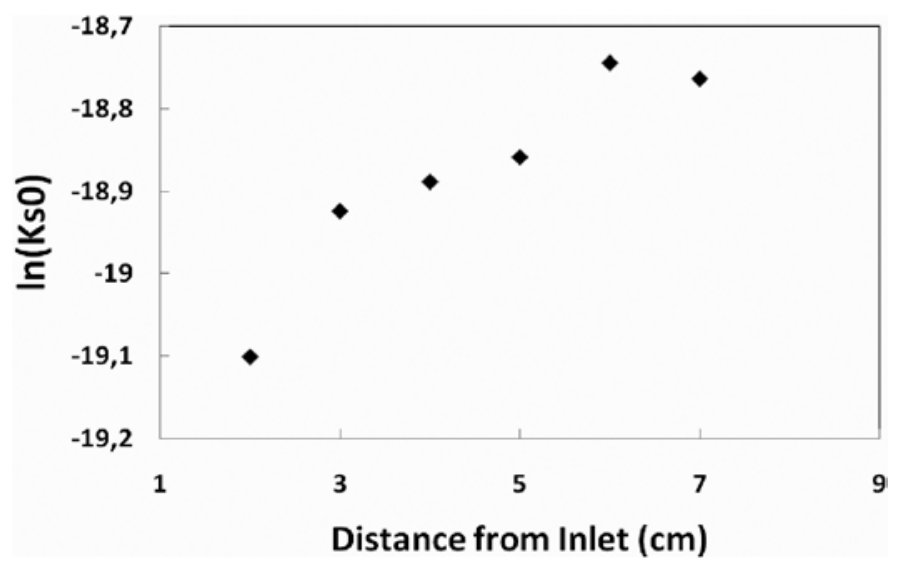

Figure 3: Estimated crystal solubility constant $(\mathrm{KsO})$ as a function of the estimated crystal surface area based on image analysis. Calculations were performed using the methods outlined by Stumm et al. [5] and Mitchell and Ferris [2]. 


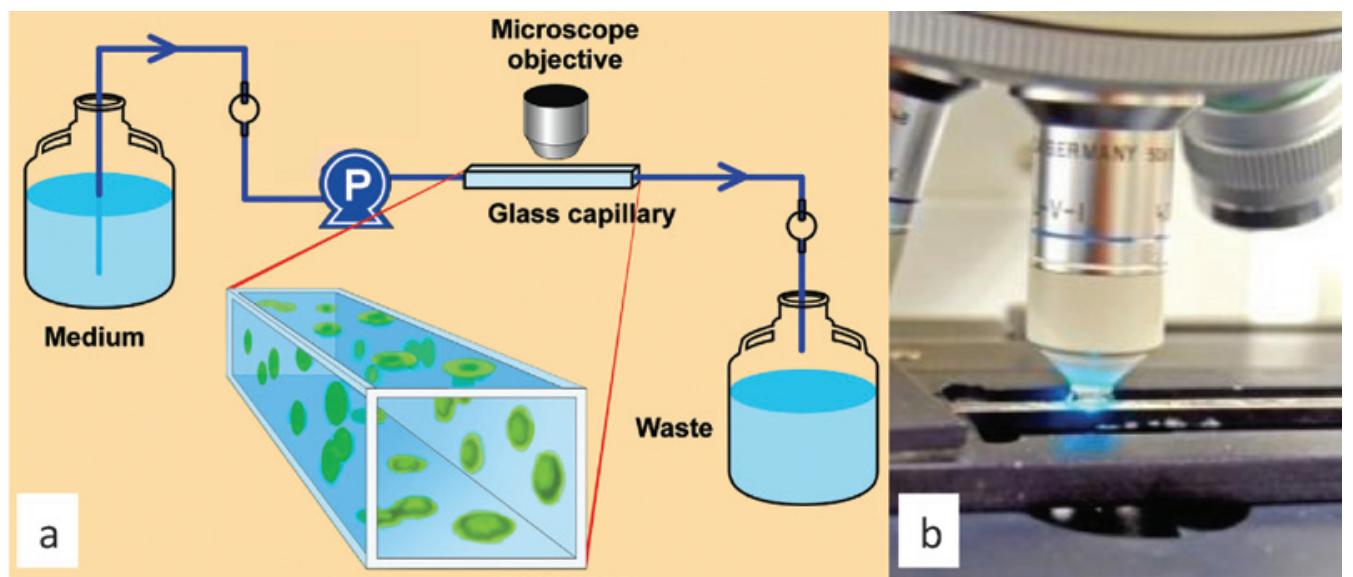

Figure 4: (a) Illustration of a $1 \mathrm{~mm}$ square glass capillary flow-cell system. Minerals and bacteria are imaged through the glass ceiling of the capillary. Drawing by J. Meyer, MSU-CBE [6]. (b) Photograph of the reactor being imaged.

DNA stains SYTO-9 (green) and propidium iodide (red). According to Invitrogen, SYTO-9 can penetrate intact cell walls and stain the DNA whereas propidium iodide cannot penetrate intact cell walls of live cells and thus indicates "dead" cellular material.

Figure $6 a$ reveals the formation of small precipitates alongside attached bacteria, and Figure $6 \mathrm{~b}$ reveals the surface topography of a precipitate attached to the glass surface. Very few cells were visible between the

attached to the glass surface of the capillary flow cells are surrounded by bacterial cells and extracellular polymeric substances.

A second study was conducted with the goal of visualizing the calcite surface topography in addition to the bacteria. This was achieved by overlaying a series of images taken in confocal reflection contrast mode with a series of fluorescent CLSM images. In this experiment, Invitrogen Corporation's LIVE/ $\mathrm{DEAD}^{\circledR}$ stain was used, which consists of the fluorescent

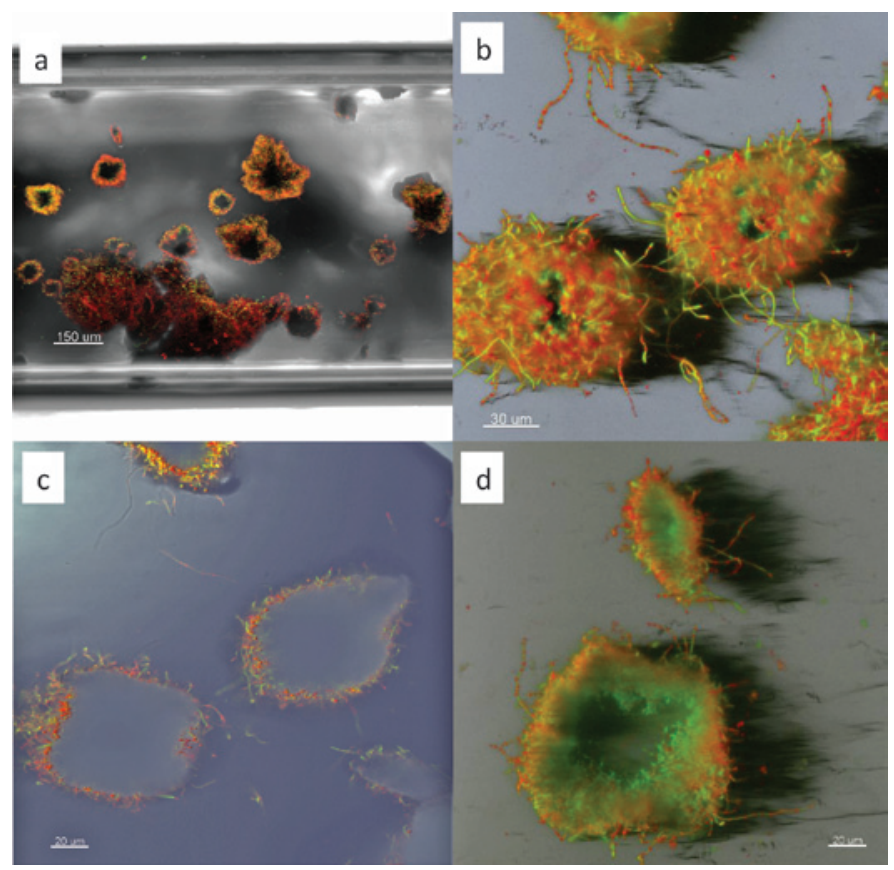

Figure 5: CLSM images of capillary stained with SYTO-11 (green) and C2d (red). (a) Image of flow cell highlighting the intimate relationship between attached cells and calcite surfaces. (b) $3-D$ reconstructed image of the perspective from inside the capillary showing cells and cell matrix surrounding calcite crystals. (c) 2-D CLSM image slice highlighting a cross section at the interior of precipitates. (d) 3-D reconstructed image of cells and cell matrix from the perspective of outside of the capillary. Only the outside of these clusters is touching the glass, and the apparent void space in the center is occupied by calcite crystals, which were not visualized in this image (see Figure 6 for reflection image overlays illustrating the presence of calcium carbonate deposits). Scale bars are 150, 30, 20, and $20 \mu \mathrm{m}$ for a, b, c, and d, respectively. mineral and the glass surface, suggesting that cells were either absent from this region or that the fluorescent stains were
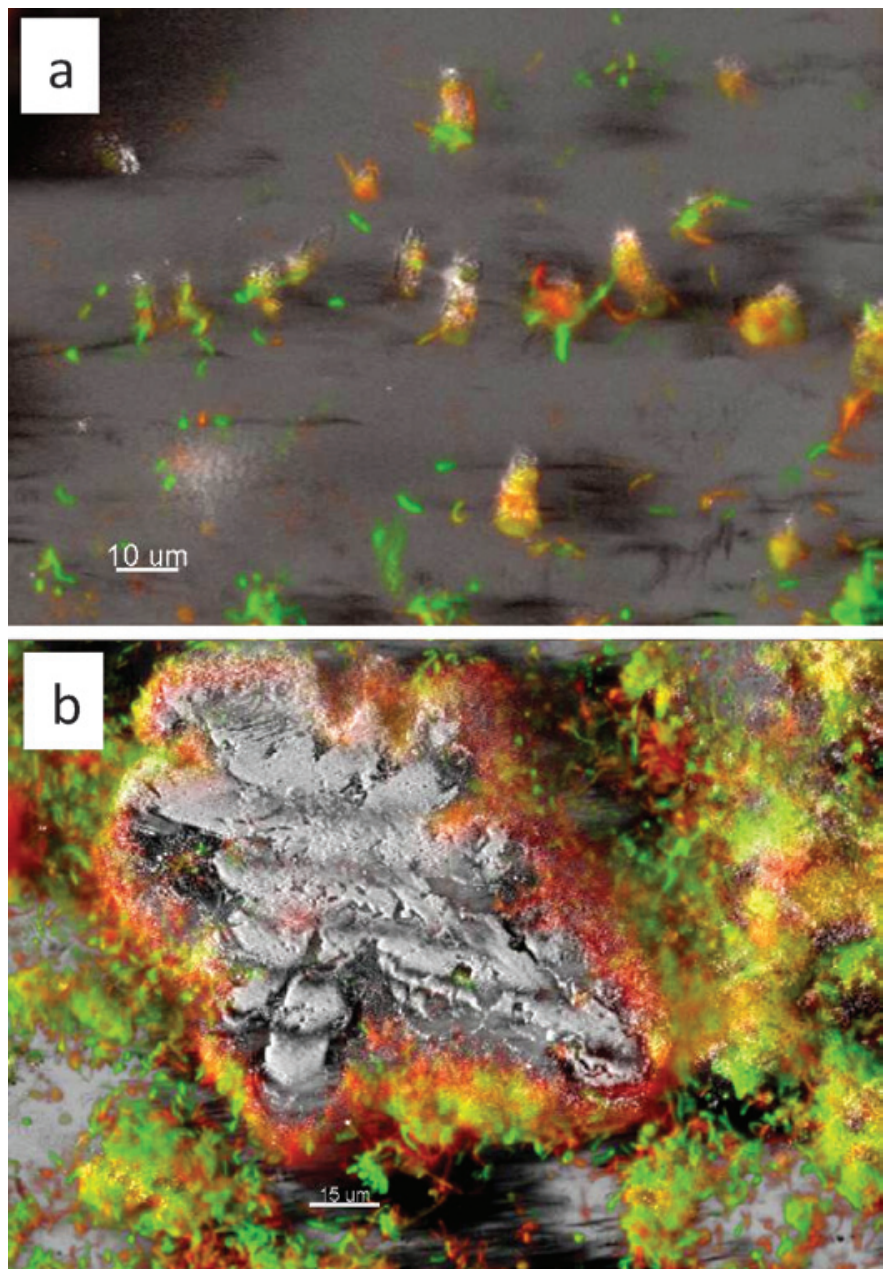

Figure 6: (a) CLSM image of a capillary stained with SYTO-9 (green) and propidium iodide (red). A reflection contrast mode stack is overlaid, showing small precipitates in close proximity to cells. (b) CLSM image from the perspective of outside the capillary. The overlaid reflection image indicates the surface topography of a large precipitate attached to the glass, and the fluorescent stains show the attached cells and cell matrix surrounding the precipitates. Scale bars are 10 and $15 \mu \mathrm{m}$ for $\mathrm{a}$ and $\mathrm{b}$, respectively. 
unable to penetrate these regions. There appears to be a higher proportion of red (propidium iodide) stained "dead" cellular material associated with the calcite crystal surface and the bottom of the biofilm proximal to the glass surface in Figure $6 \mathrm{~b}$. This suggests that solute transport in these environments might be limited, possibly because of the development of regions with low solute permeability or biological uptake of nutrients by the biofilm above.

\section{Discussion}

These studies have highlighted the usefulness of microscopic techniques at two different scales to better understand the process of biomineralization quantitatively and qualitatively.

Stereomicroscopy was used to analyze the size and distribution of calcite precipitates, the overall immobilization of dissolved calcium, and the solubility of precipitates as a function of position. The limitations of resolution (that is, not accounting for the smallest crystals) in addition to the limited ability to quantify the most densely precipitated inlet region led to an underestimate of overall calcium immobilization compared to a solution chemistry-based estimate. However, the benefits of this in situ method to quantify mineral deposition and substrate conversion with respect to the location along the flow path are significant. Controlling the spatial distribution of calcium carbonate precipitates can provide an invaluable tool for the development of strategies to manipulate porous media permeability and reactive transport on large scales. For example, during soil stabilization [1], contaminant co-precipitation [2], and geologic carbon sequestration $[7,8]$ it will be important to either promote or prevent biomineral clogging at a certain distance from the injection point.

The groundbreaking confocal scanning laser microscopy work by Pitts and Stewart [6] demonstrating the power of observing biofilms in-situ using CLSM was expanded in this study to include biomineral formation. The findings of this paper show that mineral surfaces can provide a suitable environment for microbial attachment and that the effect of bacteria may extend beyond the alteration of bulk fluid chemistry (for example, ureolysis). Further, attached microbial communities may have significant local effects on the chemical, biological, and nucleation environment at the mineral surface.

\section{Future Work}

Ongoing investigations are seeking to gain a better understanding of the association of bacteria throughout the process of precipitate formation. For instance, it is unclear at this point whether cells attach prior to mineral nucleation or if calcium carbonate precipitates form prior to bacterial attachment on the mineral surface. Additional experimental work will also focus on understanding the distribution of cells in the interior of the mineral precipitates.

\section{Acknowledgments}

This work was supported by the U.S. Department of Energy (DOE) Zero Emissions Research Technology (ZERT) program, award no. DE-FC26-04NT42262; the DOE Office of Science, Environmental Remediation Science Program (ERSP), contract no. DE-FG02-09ER64758; the U.S. Department of Energy EPSCoR program under grant no. DE-FG02-08ER46527; and the National Science Foundation Award No. DMS-0934696. The authors also acknowledge funding for the establishment and operation of the Environmental and Biofilm Mass Spectrometry Facility at Montana State University through the Defense University Research Instrumentation Program (DURIP, Contract Number: W911NF0510255) and the MSU Thermal Biology Institute from the NASA Exobiology Program (Project NAG5-8807). Imaging facilities were provided by the Center for Biofilm Engineering, Bozeman, MT, and the confocal microscope was funded through a grant from the M.J. Murdock Charitable Trust.

\section{References}

[1] V Whiffin, L van Paassen, and M Harkes, Geomicrobiol J 24 (2007) 417-23.

[2] A Mitchell and G Ferris, Environ Sci Technol 40 (2006) 213-26.

[3] G Ferris, L Stehmeier, A Kantzas, and F Mourits, J Can Petrol Technol 13 (1996) 57-67.

[4] W Rasband, ImageJ, U.S. National Institutes of Health, Bethesda, Maryland, USA. http://rsb.info.nih.gov/ij/. 1997-2009.

[5] W Stumm and J Morgan, Aquatic Chemistry, Wiley, New York, 1996, p. 1022.

[6] B Pitts and P Stewart, Microscopy Today 16(4) (2008) 18-22.

[7] A Mitchell, K Dideriksen, L Spangler, A Cunningham, and R Gerlach, Environ Sci Technol 44 (2010) 5270-76.

[8] A Cunningham, R Gerlach, L Spangler, and A Mitchell, Energy Procedia 1 (2009) 3245-52.

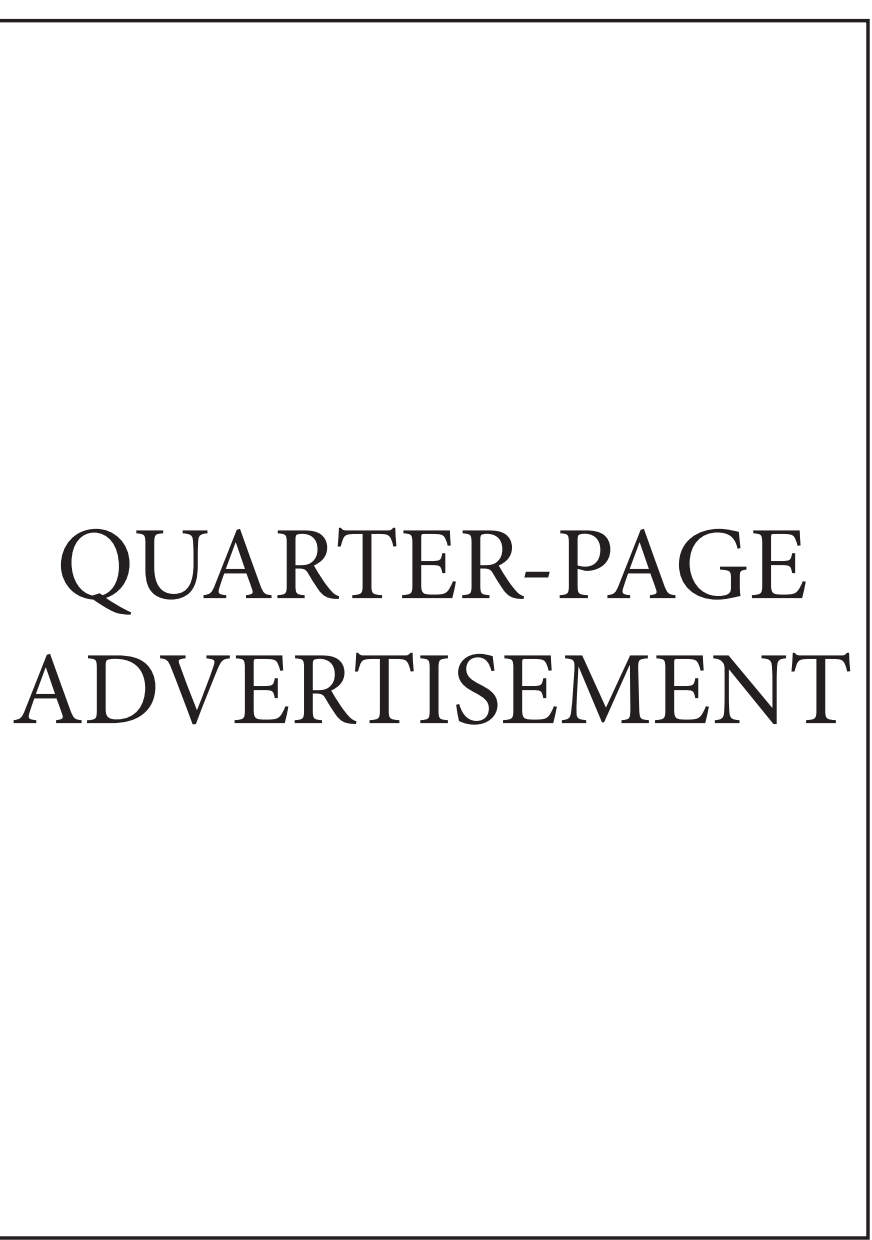

